\title{
Incidencia del conflicto armado en la vida de estudiantes universitarios en Colombia
}

William Rodrigo Avendaño-Castro

orcid.org/0000-0002-7510-8222

Universidad Francisco de Paula

Santander, Colombia

williamavendano@ufps.edu.co
Héctor Fernando Gómez-Goyeneche

orcid.org/0000-0001-7295-3589

Clínica del Country

Bogotá, Colombia

\section{Anny Julieth Triana-Orobajo}

orcid.org/0000-0002-5609-4419

Servicio Nacional de Aprendizaje

(SENA), Colombia

ajtrianao@misena.edu.co

\section{Resumen}

El objetivo de este artículo es reconocer la incidencia del conflicto armado interno colombiano en las decisiones de los universitarios, relacionadas con sus actividades curriculares, de investigación o extensión. La investigación se sustenta en el paradigma empírico analítico y el enfoque cuantitativo, con un diseño no experimental, transversal y descriptivo. Se diseñó un cuestionario a partir de entrevistas previas a personal de los departamentos de Bienestar Universitario, de dos universidades seleccionadas; y se aplicó a una muestra intencionada de 684 estudiantes. Aunque el conflicto incide en algunas decisiones sobre actividades académicas o proyectos de investigación de los universitarios, no está presente de manera significativa en la reflexión y las actividades académicas de las universidades.

\section{Palabras clave (Fuente: tesauro de la Unesco)}

Conflicto armado; estudiante universitario; formación universitaria; universidad. 


\title{
Impact of the Armed Conflict on the Life of University Students in Colombia
}

\begin{abstract}
The aim of the article is to recognize the impact of Colombian internal armed conflict on the decisions of university students in relation to their curricular, research or extramural activities. The research is based on the analytical empirical paradigm with a quantitative approach and a non-experimental, cross-sectional, descriptive design. A questionnaire was designed using previous interviews with personnel from university welfare departments of the two selected universities and administered to a purposive sample of 684 students. Although the conflict affects some decisions of university students on their academic activities or research projects, it is not significantly present in the thought and academic activities of universities.
\end{abstract}

\section{Keywords (Source: Unesco Thesaurus)}

Armed conflicts; Colombian armed conflict; university education; higher education; curriculum; university research; Colombia. 


\section{Incidência do conflito armado na vida de estudantes universitários na Colômbia}

Resumo

O objetivo deste artigo é reconhecer a incidência do conflito armado interno colombiano nas decisões dos universitários referentes a atividades curriculares, de pesquisa ou de extensão. A pesquisa foi baseada no paradigma empírico analítico e na abordagem quantitativa, e teve um desenho não experimental, descritivo e transversal. Um questionário foi desenhado a partir de entrevistas anteriores com pessoal de departamentos de bem-estar universitário das duas universidades selecionadas, e foi aplicado a uma amostra intencional de 684 alunos. Embora o conflito armado interno afete em algumas decisões sobre atividades acadêmicas ou projetos de pesquisa dos universitários, não está presente de maneira significativa na reflexão e nas atividades acadêmicas das universidades.

\section{Palavras-chave (Fonte: tesauro da Unesco)}

Conflito armado; conflito armado colombiano; educação universitária; ensino superior; plano de estudos; pesquisa universitária; Colômbia. 


\section{Introducción}

La universidad tiene por objeto formar de manera integral a los individuos para que estos puedan responder adecuadamente a las exigencias y retos del entorno en el que viven. $Y$ no se trata de una tarea sencilla, pues el mundo actual requiere de personas capacitadas para interactuar y desenvolverse en muy diversos escenarios: laboral, ciudadano, político, económico y académico, entre otros. Como lo describe Larraín y González: "la formación universitaria no solo está ligada a lo laboral, sino que, como toda educación formal, debe preocuparse de la persona en su integridad, como un ser en desarrollo y como sujeto social" (2005, p. 13). En ese orden de ideas, el contexto sociopolítico colombiano evidencia elementos particulares que las universidades no pueden desconocer. Tal vez la característica más prominente del territorio colombiano es su conflicto armado interno, que ha tenido una vigencia de más de 50 años. Por ello, este ofrece un marco de reflexión para las universidades, que no pueden ser ajenas a esta realidad que influye de forma directa e indirecta en todos los habitantes y que puede tener una incidencia significativa en la misma comunidad universitaria, aunque sin reconocer aún sus verdaderas proporciones.

Uno de los principales retos de la educación es proponer medios, estrategias y vías alternativas para fortalecer las relaciones pacíficas y la convivencia ciudadana. En efecto, la educación no solo se ubica en el ámbito intelectual, sino que está llamada a atender las necesidades integrales de los actores con los que se relaciona (Zurbano, 1998). En el espectro sociopolítico, la educación para la paz representa uno de los temas más importantes dentro de la literatura académica y ello se debe a que el actual contexto social está marcado por un ciclo de violencia, que se ha incorporado y asimilado en las representaciones e imaginarios de los sujetos, con la consiguiente adaptación de los individuos a este fenómeno.

La educación tiene un papel reedificante en la sociedad, porque puede cambiar las mentes y los sentimientos que enajenan a aquellos que participaron en el conflicto, y por ello se deben aplicar enfoques teóricos y epistemológicos que realmente puedan ser usados en la práctica para que el binomio educación y conflicto sea investigado más allá del lente teórico de la paz (Paulson y Rappleye, 2007). Una mirada reflexiva, crítica y alternativa al fenómeno que se centre en la educación universitaria solo puede ser definida en la medida en que se identifique el impacto o la incidencia del contexto del conflicto armado en la vida de los estudiantes universitarios.

Existe una doble relación entre los conceptos educación universitaria y conflicto armado: por un lado, la educación superior se puede interpretar como un mecanismo de transformación y un dispositivo de poder de naturaleza simbólica para la construcción de cambios sociopolíticos, con potencial para mitigar la reproducción de las formas de violencia vinculada con el conflicto armado, $y$, por otro, la educación se encuentra influenciada de múltiples formas por los efectos del conflicto, ya sea por su incidencia directa en los actores educativos (autoridades públicas, directivos, docentes y estudiantes) o por sus repercusiones en el sistema educativo en general.

En el primer caso, estas relaciones se ven claramente desarrolladas e identificadas en los estudios, investigaciones e informes disponibles en la literatura especializada', cada uno de los cuales explica el potencial de la educación como parte de la estrategia para transformar las sociedades marcadas por el conflicto armado y la violencia e invita a las instituciones y entidades relacionados con la educación a que generen estrategias, programas y acciones que faciliten una formación basada en la ciudadanía, la convivencia, la paz y la apertura de espacios de diálogo.

$1 \quad$ Entre otros: Nasi y Rettberg (2005); Delgado (2010), Infante (2013), Pérez (2014), Márquez (2014), Echavarría, Bernal, Murcia, González y Castro (2015), Barreto (2015), Baquero y Ariza (2014), Restrepo (2013). 
Sin embargo, el segundo enfoque no cuenta con un desarrollo científico significativo, pues no existen en la literatura modelos, enfoques o teorías que expliquen la incidencia del conflicto armado en los estudiantes de pregrado. En este terreno se ven algunos estudios que explican la forma en que los estudiantes son amenazados por el conflicto armado de manera directa, ya sea por intimidaciones, riesgos de lesiones, violencia sexual, reclutamiento forzado, desplazamiento, destrucción de instalaciones, o por otras vías. Pero se ha dejado de lado al estudiante, que carga en sus representaciones sociales e imaginarios de dicho fenómeno, los cuales inciden en sus decisiones académicas.

En esta investigación se buscó reconocer la incidencia del conflicto armado interno colombiano en la vida universitaria de estudiantes de pregrado partiendo del caso de dos universidades en Colombia y las decisiones estudiantes de pregrado respecto de actividades curriculares, de investigación o de extensión. De este modo, el estudio busca reconocer esas relaciones entre educación superior y escenarios de conflicto y ver cómo impactan la vida universitaria (Novelli y López, 2008, p. 2). En efecto, se requiere observar con mayor detalle la vida de los estudiantes que se encuentran afectados por la guerra, porque la tarea de comprensión e interpretación del conflicto se enriquece si se reconoce la incidencia del mismo en la cotidianidad (Girard y Koch, 2001, p. 47).

Para lo anterior, de las dos universidades seleccionadas, primero se caracterizaron las decisiones de los estudiantes, como consecuencia de haber sido afectados por el conflicto armado; después se identificaron algunas lecciones aprendidas por los estudiantes de pregrado como producto de dichas decisiones; y por último se estableció la medida en que la universidad ha dado respuesta a esas afectaciones en los ámbitos del quehacer universitario curricular o de aprendizaje, investigativo y de extensión.

La investigación partió de la hipótesis de que el conflicto interno colombiano ha tenido una inci- dencia multidimensional en la vida universitaria de los estudiantes, pues los efectos pueden ser localizados en las representaciones, imaginarios, decisiones, elecciones, acciones, discursos y posturas manifestadas respecto del mundo y la realidad que los circunda. Como lo expresan Baquero y Ariza (2014, p. 116), los estudiantes en Colombia han aprendido el significado de la palabra guerra no solo por lo que escuchan y debaten dentro de la universidad, sino, además, por las incidencias del fenómeno en sus vidas personales.

\section{Marco teórico}

\section{El conflicto armado interno colombiano $y$ victimas}

Determinar la naturaleza del conflicto en general no es fácil, debido a la multiplicidad de situaciones en las que se puede configurar, los distintos intereses involucrados, la variedad de los participantes o actores, así como de las posiciones y el poder que tienen, entre otros tantos factores. Siendo una condición que hace parte del ser humano, en las universidades se presenta como un elemento ausente del currículo (Girard y Koch, 2001, p. 45). Aun así, las universidades pueden visualizar el conflicto en su más amplia expresión como un medio para llevar a cabo estrategias, planes y acciones que conduzcan a transformaciones significativas. El conflicto presenta múltiples definiciones en la literatura especializada (Fisas, 1998; Salm (2006), así como una amplia tipología, dentro de la cual se encuentran los conflictos armados donde convergen la violencia directa, la violencia estructural y la violencia cultural (Fisas, 1998).

De acuerdo con Nieto (2004, p. 32), un conflicto armado no es, stricto sensu, una guerra, razón por la cual se ha escogido ese término (conflicto armado interno) para diferenciarlo de aquella. Los conflictos armados se refieren a los enfrentamientos entre fuerzas del Estado y otros grupos denominados rebeldes, insurgentes o irregulares (Díaz 
y Sánchez, 2004, p. 21; Cañadas et al., 2006, p. 17). El conflicto armado interno es una categoría de los conflictos armados y hace referencia a los enfrentamientos entre el Estado y grupos de oposición interna, sin la participación de otros Estados en apoyo de uno u otro bando (Pizarro, 2004, p. 39). Las teorías que intentan identificar los orígenes de los conflictos se enfocan básicamente en cuatro componentes: las condiciones de pobreza económica, los sistemas políticos represivos, la degradación de los recursos y la diversidad étnica (Smith, 2000, p. 6).

Respecto del conflicto armado interno colombiano, se tiene que sus orígenes aún no son precisos. Para algunos, se debe analizar el pasado remoto y próximo de la historia del país para identificar los factores asociados con los periodos de violencia, y otros consideran que dichos factores se pueden encontrar en momentos históricos precisos (Pizarro, 2015, p. 8). De esta manera, algunos autores ubican el origen a mediados del siglo XX y otros se extienden al siglo XIX, cuando se daban los procesos de construcción del Estado nación. Las dinámicas del poder político marcadas por el bipartidismo influyeron de manera profunda en la violencia en los primeros 50 años del siglo XX en el país. Además de ello, se consideran otros elementos o factores de orden externo que incidieron en la violencia, como la aparición del comunismo.

El conflicto armado en el país ha sido caracterizado de forma amplia en las últimas décadas por varios expertos en investigaciones que buscan explicar las dinámicas históricas, sociales, políticas, económicas y culturales que enmarcan el fenómeno (De Zubiría, 2015; Pizarro, 2015; Giraldo, 2015; Torrijos, 2015). En los estudiosos sobre el tema se dan posiciones encontradas, lo cual ha conducido a un amplio debate jurídico y académico. Se puede afirmar que estas realidades y manifestaciones deben ser objeto de la misma educación y la pedagogía, pues el contexto sociohistórico y político delimita la realidad de los niños y jóvenes del país y, por tanto, de la misma acción docente. De allí que resulte trascendental las investigaciones que tienen por propósito explicar las relaciones entre conflicto armado y educación, en los más diversos niveles y desde las diferentes aristas y perspectivas.

La caracterización del conflicto armado que hacen los autores es variada. Para algunos se trata de un conflicto social armado y para otros es una guerra civil o simplemente una guerra. Giraldo (2015), Pizarro (2015) y Torrijos (2015) encuentran que el conflicto armado interno se ha caracterizado por ser:

1. Prolongado, pues inicia desde La Violencia de mediados de siglo o antes y se recrudece en los años 80 .

2. Complejo, por los múltiples actores involucrados: fuerzas del Estado, guerrillas, paramilitares y ahora, aunque no tienen el estatus de actores del conflicto armado, las Facrim (bandas criminales disidentes de las Fuerzas Armadas Revolucionarias de Colombia - FARC) y las Bacrim (bandas criminales), estas últimas con presencia en 189 municipios en 2012 (FIP, 2015).

3. Discontinuo, debido a que las guerrillas de primera generación fueron debilitadas en el periodo del Frente Nacional y con posterioridad se fortalecieron en la década de los 70, evidenciando periodos caracterizados por diferentes elementos.

4. Con enormes diferencias regionales, lo cual se traduce en características diversas del conflicto, dependiendo de los espacios geográficos.

5. Con graves repercusiones en la población civil.

6. Con raíces políticas, dado el antagonismo ideológico de sus actores.

Tratándose de un fenómeno tan prolongado en el tiempo y el espacio, la población civil ha sido la principal afectada con ocasión de las dinámicas del conflicto y la ausencia/inoperancia del Estado. Las víctimas son una figura de vital importancia en la 
esfera del conflicto, tanto así que se ha considerado como un epifenómeno del conflicto y pueden adquirir diferentes tipologías o categorías.

Torrijos (2015), basado en los instrumentos internacionales de las Naciones Unidas y los pronunciamientos de la Corte Constitucional en varias sentencias, caracteriza así a la víctima:

Toda persona que haya sufrido daños, individual o colectivamente, incluidas lesiones físicas o mentales, sufrimiento emocional, pérdidas económicas o menoscabo sustancial de sus derechos fundamentales, como consecuencia de acciones $u$ omisiones que constituyan una violación manifiesta de las normas internacionales de derechos humanos o una violación grave del derecho internacional humanitario definición a la que queda integrada la familia inmediata o las personas a cargo de la víctima directa y las personas que hayan sufrido daños al intervenir para prestar asistencia a víctimas en peligro o para impedir la victimización. (p.32)

Este mismo autor divide a las víctimas en conformistas y restauradoras. Las primeras son "aquellas que simplifican su realidad en torno a la capacidad individual de perdonar a sus victimarios y olvidar o ignorar tanto los padecimientos sufridos como los que sigue padeciendo la sociedad", y las segundas son aquellas que muestran responsabilidad con ellas mismas y con los demás, y por ello se preocupan por reparar y reconstruir el tejido social y por proteger los valores de la democracia. Pero Torrijos va más allá en la construcción de una noción comprehensiva de víctima y señala que hay un fenómeno que puede denominarse "percepción de victimización transversal", el cual se traduce en el "clima social en que vive la mayoría de ciudadanos de un país y que se caracteriza por la convicción de que han padecido daños intencionados, inmerecidos, injustos, inmorales y con penosas consecuencias provocados por unas agrupaciones armadas ilegales" (p. 34).
En consecuencia, existe un concepto amplio de víctima, pues no solo se trata de un hecho jurídico, sino de una experiencia subjetiva. Por tanto, no son víctimas, para efectos de esta investigación, solo aquellos que por disposición legal reúnen los requisitos trazados en el ordenamiento jurídico, sino todos aquellos que desde su experiencia y vivencias han sido afectados por el amplio conflicto armado, incluidos otros perpetradores, como agentes del Estado que actúan por fuera de sus funciones constitucionales y legales o las Bacrim. Sin embargo, también se considera una tipología de victimas según las formas de victimización o los hechos victimizantes que se incorporan dentro de la Ley 1448 de 2011: homicidio, desaparición forzada, secuestro, lesiones personales que generaron incapacidad permanente o discapacidad, lesiones personales que generaron incapacidad, reclutamiento ilícito de niños, niñas y adolescentes, delitos contra la libertad e integridad sexual, incluidos niños, niñas y adolescentes nacidos como consecuencia de una violación sexual en el marco del conflicto armado, tortura, tratos crueles, inhumanos o degradantes; desplazamiento forzado y despojo.

\section{El proceso en la toma de decisiones}

Esta línea teórica conceptual permite reconocer que los estudiantes universitarios demuestran en las decisiones adoptadas una posición respecto de su realidad, es decir, frente a las afectaciones y experiencias de vida, para un enriquecimiento personal y la construcción de mejores escenarios para el mismo sujeto y para los que lo rodean. Dos perspectivas permiten comprender este fenómeno: la teoría de la elección racional y el enfoque tomista, en cuanto a acto y potencia. De acuerdo con Vidal (2008, p. 222), la teoría de la elección racional es "una perspectiva teórica general de las ciencias del comportamiento humano, y su ámbito es el de la interacción humana, es decir, se refiere a toda clase de situaciones sociales", dentro de las cuales encajaría el fenómeno que se estudia. Se puede sintetizar en el siguiente enunciado: "cuando enfrenta varios cursos 
de acción, la gente suele hacer lo que cree que es probable que tenga mejor resultado general" (Elster, 1995, p. 31). Desde esta perspectiva, una persona, frente a diversas alternativas de elección, optará por aquella que considere que tendrá el mejor beneficio o resultado (Miklos, 2001, p. 171).

Se debe resaltar que la teoría en mención tiene un carácter instrumental, esto es, que atiende al resultado externo que se obtiene con la decisión tomada. Y esto se debe a que la decisión conduce a la obtención de un resultado y guía la acción del individuo. Desde esta perspectiva, el curso de la acción y el resultado movilizado por la decisión son el producto de un proceso racional en el cual se establecen criterios de ordenamiento de preferencias (proceso jerarquizador). Se parte de la hipótesis de que el sujeto cuenta con una información, unos objetivos y con unos elementos subjetivos que condicionan la decisión y la acción (v.gr. recursos y capacidades) (Miklos, 2001).

La decisión comporta "elegir el mejor de los cursos de acción, dados los objetivos y las restricciones del sujeto" (Miklos, 2001, p. 172). En otros términos, la decisión corresponde a una elección que determina la acción del individuo, y ello depende de los objetivos, las restricciones objetivas y la percepción sobre la utilidad que tiene cada una de las alternativas. Conforme a Miklos, para que se pueda llegar a una elección racional se requiere: "a) acuerdo total sobre los objetivos, b) conocimiento exhaustivo de todas las opciones para obtener el objetivo, c) disponibilidad plena de recursos (tiempo, personal, dinero y flexibilidad institucional) para conseguir el objetivo, y d) las estrategias adoptadas para conseguir el objetivo, las cuales se basan en una relación fluida entre fines y medios" (p. 172).

En el contexto de esta investigación, las decisiones tomadas por los estudiantes reflejarían una incidencia manifiesta del conflicto armado, que los lleva a buscar las alternativas más favorables para contribuir a la solución del problema que enfrentan.
Sin embargo, no todas las decisiones tienen un curso o propósito instrumental, porque pueden contribuir al crecimiento personal, aunque el agente no hubiera obtenido el resultado que buscaba.

Ahora bien, el enfoque tomista de acto y potencia permite comprender la manera como las decisiones ejecutadas demuestran la motivación de las personas -en este caso, resolver las afectaciones del conflicto- $y$ los efectos positivos que pueden tener en el individuo que toma decisiones. Se entiende que los estudiantes objeto de la investigación mantienen un conjunto de cualidades que determinan su individualidad y los hace particulares. Dichas características inciden en el mismo acto, pues en el comportamiento está involucrado todo su ser, esto es, la esencia. En consecuencia, las decisiones que se toman son una manifestación no solo del efecto del conflicto armado, sino que además son fuentes de lecciones y de enriquecimiento personal que logran modificar la esencia misma del individuo.

Entonces, se entiende que los estudiantes que han sido afectados por el conflicto armado interno al momento de tomar decisiones están llevando a cabo una operación que no se consuma ni se extingue en la acción, sino que se mantiene de manera indefinida e involucra todo el ser del individuo (García, 2000). Como ya se ha señalado, esas decisiones generan un acto, una operación de amplia extensión y alcance que modifica al individuo, lo enriquece, lo transforma, lo modifica en su esencia.

En definitiva, la mirada metafísica de las decisiones nos sugiere que, más allá de las afectaciones sufridas por la persona o los cursos tomados para enriquecer un saber intelectual, la toma de decisiones es la manera como la persona enriquece su propio ser personal. En el contexto universitario, esto quiere decir que los diferentes ámbitos de desempeño y formación se convierten en oportunidad real para actualizar el potencial de crecimiento humano a fin de extraer lecciones valiosas, tanto para la vida de quienes han sido víctimas como para aquellos que entren en relación con estos sujetos. 


\section{Método}

La investigación se sustenta en el paradigma empírico analítico, es de naturaleza cuantitativa y tiene un diseño no experimental, transversal y descriptivo. Se estructuro en tres etapas: 1) revisión de bibliografía, 2) diseño y validación estadística de instrumentos, y 3) sistematización, análisis e interpretación de resultados. Participaron en el estudio los estudiantes de pregrado de la Universidad Francisco de Paula Santander de Cúcuta y la Universidad Militar Nueva Granada de Bogotá, a quienes se les aplicó un cuestionario estructurado. La población del estudio correspondió a un total de 1.295 estudiantes de la Universidad Francisco de Paula Santander (43,12 \%) y 2.389 de la Universidad Militar Nueva Granada (56,82 \%). En total, participaron 684 estudiantes $-295(43,12 \%)$ y 389 (56,82 \%), respectivamente-, que corresponden a una muestra intencionada, debido a que se seleccionaron los estudiantes en porcentajes iguales por cada uno de los programas académicos ofrecidos por las universidades.

Se utilizó un cuestionario como instrumento de recolección de información, el cual se validó a través de juicio de expertos. Este cuestionario se construyó a partir de una fase previa de exploración en la que se aplicaron entrevistas a funcionarios de Bienestar Universitario y apoyo a los estudiantes de ambas instituciones de educación superior, con el objetivo de reconocer su conocimiento sobre el tema, proyectos desarrollados, estrategias realizadas o adecuaciones curriculares o del plan de estudios. Esta labor preliminar permitió establecer que ninguno de los funcionarios de tales universidades tenía claridad sobre la incidencia del conflicto armado interno colombiano en los estudiantes de pregrado y en su vida universitaria en general.

El cuestionario, además de integrar información sociodemográfica general, como edad, sexo, carrera, semestre cursado, estrato socioeconómico, incorporó otras variables, como situación de víctima, victimario, afectación del conflicto en el estudiante o en un familiar, hechos victimizantes, decisiones adoptadas en el ámbito universitario a partir de esta realidad, y, en ese mismo contexto relacional, participación en discusiones, desarrollo de trabajos académicos, participación en grupos de investigación, actividades programadas o actividades de extensión y lecciones aprendidas.

\section{Resultados}

La información fue recolectada de manera virtual a través de Google Forms, lo que permitió sistematizarla de manera automática. De esta manera, los investigadores invitaron a los estudiantes en general a desarrollar el cuestionario, hasta cumplirse con las cantidades contempladas en la muestra por programas y carreras. Una vez que se obtuvo la totalidad de la información, se hizo el respectivo análisis e interpretación de los hallazgos a la luz del referente teórico seleccionado. A continuación se muestran los resultados del estudio desarrollado, los cuales responden a los datos recolectados a través del cuestionario y a la forma de sistematización y análisis indicada.

\section{Aspectos sociodemográficos}

El 17,28 \% de estudiantes de la Universidad Francisco de Paula Santander (UFPS) señalo ser víctima del conflicto armado interno (51 estudiantes), y lo mismo señaló el 18,50 \% de los que participaron por la Universidad Militar Nueva Granada (UMNG) (72 estudiantes). Es decir, el 17,98 \% del total de la población de ambas universidades se encuentran afectados por el conflicto armado interno conforme a las formas de victimización reconocidas dentro de la tipología de las Naciones Unidas y cuya clasificación se divide en física directa y psíquica/moral directa.

La población participante no muestra una tendencia específica en cuanto al género. Mientras que en la UFPS los individuos que participaron son mujeres en un $73 \%$, en la UMNG la población estuvo dividida sin mayor diferencia (50,38\% hombres y 49,61 $\%$ mujeres). La edad de los participantes en las dos 
universidades se concentró en el rango que va hasta los 25 años, predominando en el grupo de menores de 20 años para ambas universidades $(55,93 \%$ y $57,58 \%$, respectivamente). Respecto del estrato socioeconómico, el 81,01 \% de estudiantes de la UFPS se ubican en los estratos 1 y 2 , mientras que el $71,97 \%$ de los de la UMNG se encuentran en estratos 3 y 4.

La población participante muestra dispersión en cuanto al año que cursan, en ambas universidades. Los estudiantes seleccionados se ubican a lo largo del curso de las carreras, siendo el periodo de menor porcentaje los correspondientes a los dos últimos años de la carrera. En ambos casos los estudiantes cursan, principalmente, los primeros años de la carrera: cerca del $70 \%$ para las dos instituciones sí se suman los porcentajes del primer, segundo y tercer año.

\section{Formas de victimización y victimarios predominantes}

Teniendo en cuenta las formas de victimización reconocidas por las Naciones Unidas, de los estudiantes que refieren haber sido víctimas o haber sido afectados por el conflicto armado interno en Colombia, para la UFPS se evidencia mayor porcentaje dentro de la clasificación física directa por amenazas (34 \%) y desplazamiento (24,5\%), y en la clasificación psíquica o moral directa el mayor porcentaje es dado por pérdida de oportunidades laborales, sociales y económicas (24,5\%) y afectación económica (34 \%). Para la UMNG, dentro de la clasificación física directa, igualmente se evidencia mayor porcentaje de amenazas (34,7 \%) y riesgos de lesiones físicas (16,7\%); y en la clasificación psíquica o moral directa hay un comportamiento similar para los dos grupos frente a la pérdida de oportunidades laborales, sociales y económicas (27,8 \%) y por afectación económica (25\%).

También se indagó por la afectación de familiares. Los estudiantes de la UFPS señalaron que los efectos se extendieron a los padres (73,9\%), a ellos mismos (56,5\%), a otros familiares $(56,5 \%$ ) y a hermanos y abuelos ( $47,8 \%)$. Por otro lado, los partici- pantes de la UMNG señalan que la incidencia es mayor en los padres ( $47,4 \%)$, en otros familiares $(39,5 \%)$ $y$, en menor porcentaje, en ellos mismos (28,9\%).

Respecto de los victimarios, para la UFPS, dentro de los actores que han afectado a los estudiantes en el marco del conflicto armado, se evidencia en mayor porcentaje los grupos paramilitares (56,5\%) y grupos guerrilleros $(52,2 \%)$ y, con un porcentaje importante a tener en cuenta, las Bacrim (26,1\%). Para la UMNG el comportamiento se ve reflejado con un mayor porcentaje para los grupos guerrilleros $(63,2 \%)$ y las Bacrim (28,9\%).

\section{Decisiones tomadas en respuesta a la afectación}

De los estudiantes que expresaron haber sido víctimas del conflicto armado colombiano, un bajo porcentaje decidió participar en alguna de las actividades ofrecidas por los servicios de orientación o Bienestar Universitario como consecuencia de su afectación por el conflicto armado interno. Para la UFPS se presenta el $8,7 \%$ y para la UMNG el 18,9 \%. Lo mismo sucede en otro tipo de servicios o actividades:

Servicios de ReGistro académico: Son poco utilizados por los estudiantes de pregrado. Solo el $13 \%$ de los participantes de la UFPS y el 7,9\% de los participantes de la UMNG han hecho uso de los servicios descritos. De este bajo porcentaje, los servicios que más han utilizado los estudiantes víctimas son cambio de universidad, aplazamiento de carrera, cancelación de materias y posibilidad de iniciar otra carrera para la UFPS, y aplazamiento de carrera y cancelación de materias para la UMNG.

Servicios de docencia: En la UFPS ninguno de los estudiantes afectados por el conflicto armado interno ha tomado alguno de los servicios de docencia, mientras que en la UMNG sí lo ha hecho el 31,6\%. Dentro de tales servicios, los más utilizados son: asesoría académica personalizada, programa de tutoría para becarios, jornadas universitarias y participación en seminarios y/o congresos. 
Opciones de InVESTIGACIÓn: El 34,8 \% de los participantes de la UFPS señalan haber utilizado algún tipo de opciones en este campo como consecuencia de la afectación producida por el conflicto armado, mientras que en la UMNG solo 13,2 \% manifiesta haber optado por alguna actividad en las opciones de investigación desde una perspectiva general, no necesariamente relacionada con el conflicto armado. La de mayor uso para quienes han optado por esta opción es la participación en convocatorias internas de grupos, semilleros, proyectos y/o productos de investigación.

OpCiones de extensión: Los servicios de apoyo por los que han optado los estudiantes en cada una de sus categorías (bienestar, docencia, registro, investigación o extensión) se encuentran aún en curso para el 13,2 \% de la población participante de la UMNG y para el $26,1 \%$ de la UFPS. Por otro lado, el 33,3 \% de los participantes de la UMNG que optaron por algún tipo de apoyo o servicio mantienen vínculo con las personas que se los ofrecieron, mientras que solo el $6,9 \%$ de los participantes de la UFPS mantienen vínculo con estas personas.

InICIATIVAS PERSONALES O De Voluntariado: Además de los servicios u opciones antes descritos, se encuentran las iniciativas de tipo personal o de voluntariado que han desarrollado los estudiantes como consecuencia de la afectación producida por el conflicto armado. Estos resultados son significativos por cuanto superan la participación de los estudiantes en cuanto a los servicios u opciones que han dispuesto las universidades. Para la UFPS, el $34,8 \%$ (11 estudiantes) de los participantes señalan haber tenido iniciativas personales de servicio o voluntariado y para la UMNG, el 26,3 \% (10 estudiantes) señalan lo mismo.

Las actividades académicas representan las iniciativas personales de mayor implementación de los participantes (50\% UFPS y $40 \%$ UMNG), seguida de las actividades de voluntariado (25\% UFPS y $25 \%$ UMNG). También se encuentran las actividades con

\section{Figura 1. Iniciativas personales de servicio desarrolladas por estudiantes como producto de la afectación por el conflicto armado interno}

Universidad Francisco de Paula Santander

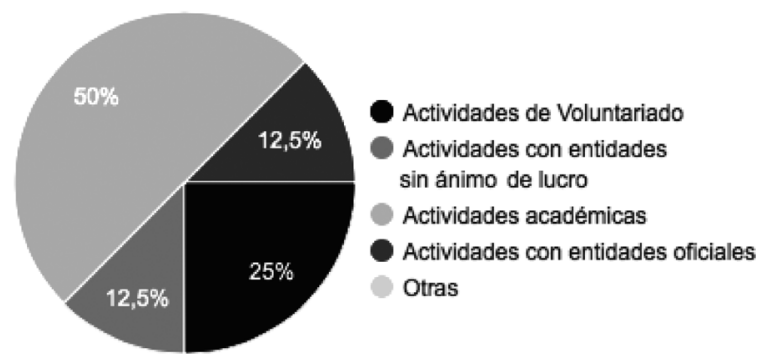

Universidad Militar Nueva Granada

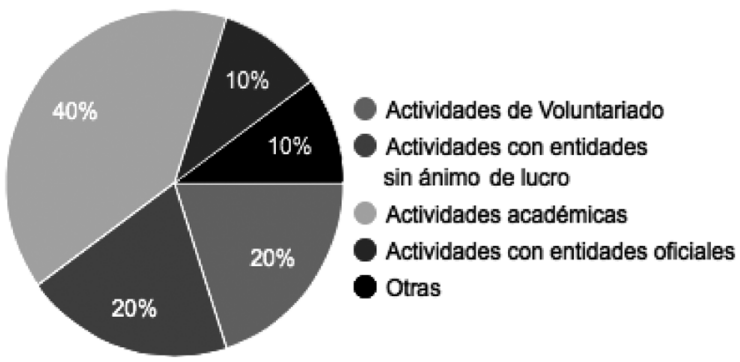

Fuente: elaboración propia.

entidades sin ánimo de lucro y actividades con entidades oficiales. Para el caso de la UMNG, el apoyo de las iniciativas ha correspondido a familiares, personas de la universidad u otras universidades, miembros de la Fuerza Pública activos o retirados, y en el $20 \%$ de los casos se ha adelantado la iniciativa de manera individual. Para el caso de la UFPS, el apoyo ha sido brindado principalmente por familiares, otras víctimas, agentes del Estado y personas de la universidad $u$ otras universidades. En el 62,5\% de los casos las iniciativas se han adelantado de manera individual y sin apoyo para el caso de esta universidad. Solo el $10 \%$ de los participantes de la UMNG continúa con las iniciativas, mientras que en la UFPS el $71,4 \%$ de los estudiantes continúa con las iniciativas mencionadas.

En relación con el apoyo que los estudiantes buscaron para el desarrollo de las iniciativas de servicio, se evidencia que, para la UFPS, ellas vinie- 
ron de sus familiares (6o \%), de ellos solos (62,5\%) y contaron con el apoyo de otras víctimas (37,5\%); para la UMNG, estas iniciativas fueron desarrolladas en compañía de sus familiares (60 \%) y personas de la propia universidad u otras universidades (30\%), entre otros apoyos, como los personales y de miembros de la Fuerza Pública (20 \% y $20 \%$, respectivamente).

\section{Las lecciones aprendidas de los estudiantes como producto de las decisiones tomadas}

Se presentaron enseñanzas, reflexiones o lecciones positivas como producto de sus decisiones para el 60,9\% del total de participantes de la UFPS y el $47,4 \%$ de la UMNG, que han optado bien por alguno de los servicios o apoyos de las universidades o bien por algún tipo de iniciativa personal.

Ahora bien, en la Tabla 1 se incluyen algunas respuestas de los participantes en cuanto a lecciones positivas aprendidas como resultado de haber tomado alguno de los servicios o apoyos de la universidad o bien por su iniciativa personal en el marco de la vida universitaria. Estas son muy variadas y se pueden ubicar especialmente dentro del campo del enriquecimiento personal, más allá de fines instrumentales.

\section{Figura 2. Reflexiones o lec ciones positivas obtenidas como producto de la participación o las iniciativas personales}

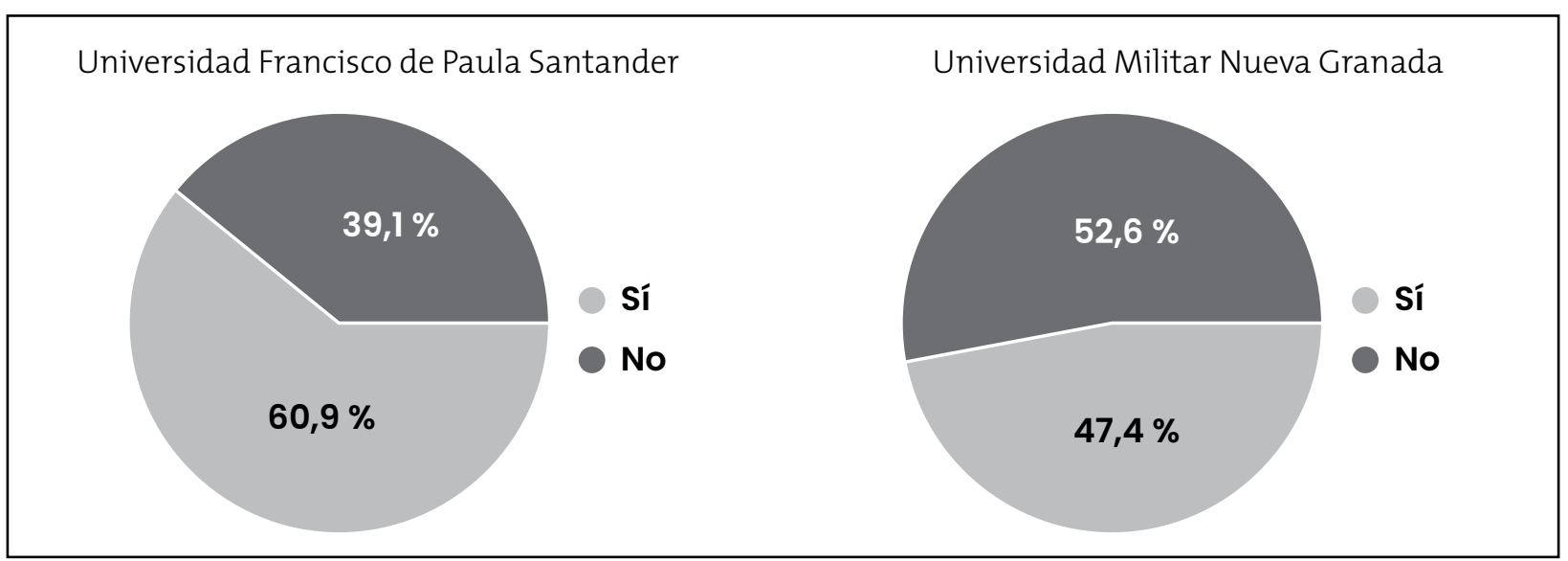

Fuente: elaboración propia.

Tabla 1. Algunas reflexiones o lecciones positivas aprendidas por los estudiantes

\section{Universidad Militar Nueva Granada}

A la hora de la verdad es más importante la familia que lo material.

Aprender a compartir y ser más tolerante.

Aprender a perdonar.

Aprovechar las oportunidades, porque solo se presentan una vez.

Buscar ayuda.

Confianza.

El apoyo de la familia.

Es algo importante para la vida. 
Es importante perdonar y ponerse en los zapatos del otro.

No importa las diferencias ni las dificultades que se sufren.

Realmente ha habido apoyo en cada uno de los entes de investigación y entes universitarios prestos al servicio de mejora continua.

Por más que las personas le quiten lo más importante en la vida, que son los padres, hay que seguir adelante.

Que la paz la iniciamos nosotros con las demás personas.

Se ha de buscar y aprovechar la ayuda de la universidad.

Aprovechar la ayuda de la universidad.

Se deben hacer más frecuentemente estas encuestas.

Se debe tomar las cosas con calma para poder enfrentar lo que está sucediendo y no apresurarse a los hechos.

\section{Universidad Francisco de Paula Santander}

La transformación social nace del trabajo hecho para el pueblo y con el pueblo, con el fin de mejorar la calidad de vida.

Con esta corta encuesta reflexioné acerca de la participación y el apoyo que se le da al estudiante víctima del conflicto armado colombiano y [vi] cómo las personas que hemos sido afectadas de manera directa por la guerra tenemos muchos beneficios, al encontrarnos en una justicia restaurativa.

Hay que ser tolerante.

La universidad nos brinda ayudas. Debemos aprovecharlas.

Soy un joven cristiano, donde he sido víctima de esas amenazas y he aconsejado a otros acercarse a Dios que Él cuida nuestras vidas y tiene el control sobre cada uno.

La vida no es fácil. Desde la escuela nos pintan una vida futura que sería color de rosa, pero nos cierran demasiadas puertas, tanto laborales como económicas y académicas.

Que la universidad tiene proyectos que desconozco para las víctimas del conflicto armado.

Está muy interesante [la encuesta] ya que nos lleva a tener una conciencia que nos lleva a la solución de aquellas situaciones vividas, con el fin de mejorar la manera de pensar y quitar esos pensamientos.

Es una actividad gratificante, ya que se puede ayudar a personas que lo necesitan; se conocen muchas historias.

Perdonar para poder gozar de la paz.

Nadie es ajeno al flagelo del conflicto armado y lo que puede producir; está en ti ayudar al cambio social.

En qué área puedo participar además de las actividades que se den dentro de la universidad.

Fuente: elaboración propia.

\section{Discusión}

Aunque el porcentaje de estudiantes encuestados y afectados por el conflicto armado interno fueron pocos: 17,28 \% para la Universidad Francisco de Paula Santander y $18,50 \%$ para la Universidad Militar Nueva Granada, los datos recolectados permiten centrar la discusión en algunos aspectos específicos y adelantar algunas reflexiones que permitan supe- rar el mero análisis cuantitativo o estadístico para hacer aportaciones al campo de estudio abordado. Esto exige que cada caso institucional sea analizado de manera diferencial, aunque, antes de dar paso a ello, se debe indicar de antemano que la incidencia del conflicto armado interno en la vida universitaria de estudiantes de pregrado se extiende a sus iniciativas 
personales, sin que encuentren en la universidad una plataforma para el desarrollo de nuevas estrategias que impulsen el trabajo proyectado, aprovechando su experiencia de vida de los participantes.

Si la vida universitaria se identifica con todas las formas y modalidades en las que pueden participar los estudiantes en el escenario de la universidad: académicas, científicas, políticas, sociales, culturales, entre otras (Trilla et. al., 2011), se puede inferir que las decisiones tomadas, y que encajan como iniciativas personales en razón de la experiencia de ser víctima del conflicto armado interno, corresponden a un elemento central de la vida universitaria. Lo importante es reconocer que los intereses e iniciativas personales de los estudiantes en el mundo universitario son formas de expresión de lo que denominamos vida universitaria, la cual se basa no solo en las actividades gestionadas por las universidades, sino, además, en las experiencias de los sujetos, así como en la imaginación, el sentimiento, la razón y la cultura, que al mezclarse son fuente de vida social (Murcia, 2009). Dicho lo anterior, se exploran e interpretan los resultados para cada uno de los casos objeto de estudio.

La Universidad Francisco de Paula Santander atiende, generalmente, población de un estrato socioeconómico bajo. Dicha realidad conlleva que los estudiantes inscritos en las diferentes carreras se encuentren en desventaja social y que muchos de ellos sean víctimas del conflicto armado interno. Por decisión de las directivas de la institución, se han formulado programas específicos de ayuda a los estudiantes que ostentan la condición de víctimas. Sin embargo, se destaca que los estudiantes de pregrado se esfuerzan más en participar o adelantar acciones inscritas en el ámbito de la iniciativa personal, más que tratarse de con del aprovechamiento de los servicios ofrecidos por la universidad. Esto implica una reflexión profunda sobre el verdadero papel de la universidad objeto de estudio, la cual se aleja de aquello que se describió en el marco de análisis de la investigación. Como ya se enfatizado, la universidad tiene como responsabilidad social acercarse al medio y re- conocer en el este las principales necesidades y problemas, para volverlos objeto de su actividad a partir de la autonomía universitaria que le corresponde.

La población estudiantil víctima del conflicto armado ha sufrido, principalmente, amenazas, desplazamiento forzado, pérdida de oportunidades sociolaborales y económicas y afectación económica, conforme a las respuestas brindadas. Guerrilla $(52,4 \%)$ y paramilitares $(57,1 \%)$ se muestran como los principales actores armados responsables de estas afectaciones. Para todos los casos, los familiares más cercanos han sido los perjudicados por las acciones de los grupos al margen de la ley señalados.

Sin embargo, los estudiantes afectados por la violencia no muestran en la mayoría de los casos incorporación a algunas de las actividades que se diseñan y ejecutan dentro de bienestar universitario. Básicamente, todos los estudiantes, a partir de su condición, aprovechan los incentivos que ofrece la universidad en materia económica o de recursos para las víctimas, pero no es tan palpable su vinculación directa con otros programas de orden cultural, psicoafectivo, de salud, deportivo o similar. Es posible que la baja participación de los estudiantes en este tipo de actividades se deba al poco interés por los servicios o programas que se dan, a la estigmatización que produce el ser víctima, o bien a que no se desea ser reconocidos como parte de este grupo. Entonces, con base en la teoría de la racionalidad, se observa que las decisiones adoptadas por este grupo de estudiantes de pregrado también se basan en la retribución, por cuanto aprovechan aquellos apoyos en materia económica o de recursos.

Ahora bien, en el terreno académico e investigativo solo algunos estudiantes señalan optar, principalmente, por la participación en proyectos de investigación o semilleros, así como en eventos de carácter académico, como congresos y seminarios. Por otro lado, algunos estudiantes muestran que, a partir de la realidad que se desprende del conflicto armado, han optado por iniciativas personales de 
servicio. Dentro de estas se hallan actividades académicas, de voluntariado, con entidades oficiales y con entidades sin ánimo de lucro. En estos casos, las iniciativas se han realizado con entidades públicas, con familiares y con otras víctimas. Resulta valioso resaltar este aspecto, porque las decisiones de los estudiantes de pregrado de participar en iniciativas personales de servicio se deben a un interés producto de su experiencia personal con ocasión del conflicto armado del cual han sido víctimas, y ello modifica su existencia e integridad como seres humanos.

En la mayoría de los casos donde se da una participación más activa de los estudiantes, las actividades desarrolladas han generado un efecto positivo. Algunos estudiantes refieren que las actividades en las que ha participado las acercan más a otras personas afectadas: "mayor empatía hacia las diferentes personas afectadas", "hay que ser más tolerantes". Para otros, la universidad brinda oportunidades, pero estas no son conocidas y las interpretan como un beneficio que podría ser aprovechado. Y otros consideran que la labor que se pueda realizar en la universidad puede ser una oportunidad para generar un cambio social y personal profundo. Aunque son pocos los estudiantes que deciden vincularse de manera directa con actividades o programas académicos, investigativos, culturales y deportivos, se observa que los mismos reconocen más ampliamente la necesidad de generar acciones de cambio social, pues ven que la violencia deja secuelas serias y significativas en las personas.

Ahora bien, en cuanto a la Universidad Militar Nueva Granada, se encontró que los estudiantes afectados por el conflicto armado interno han sufrido, principalmente, los siguientes hechos: amenazas, pérdida de oportunidades sociolaborales y económicas, afectación económica, riesgos de lesiones físicas. Guerrilla, bandas criminales y paramilitares aparecen como los principales actores responsables de estas afectaciones. Familiares más cercanos a estos estudiantes también han sido afectados por las acciones de los grupos al margen de la ley.
Al igual que en la otra institución, un bajo porcentaje de estudiantes participa en las actividades de Bienestar Universitario y las dependencias adscritas a la misma y quienes hacen uso de estos servicios de apoyo se integran, generalmente, a las actividades culturales, deportivas y de asistencia psicológica. En el terreno del apoyo docente, un grupo, aunque no significativo, busca principalmente la asesoría académica y las tutorías. La participación en actividades de investigación tampoco resulta muy habitual entre los estudiantes afectados por la violencia y los que deciden participar se centran en las convocatorias a proyectos de investigación, semilleros o aquellas convocatorias externas. También se observa pocos estudiantes que deciden efectuar alguna iniciativa personal de servicio y los que lo hacen se inclinan principalmente por las actividades académicas, de voluntariado y con entidades sin ánimo de lucro.

Dentro de los aspectos positivos que exaltan los estudiantes como producto de sus decisiones se encuentran: tolerancia y capacidad de reconciliación, mayor aprovechamiento de la oferta de la universidad, mejores oportunidades para la reconstrucción del tejido social y mayor comprensión de la realidad a partir de las actividades de investigación. Estas lecciones aprendidas van más allá de una posible retribución y redundan en el enriquecimiento personal como principal motivación por la que deciden participar en tales actividades.

En la Universidad Francisco de Paula Santander hay mayor participación de los estudiantes de pregrado víctimas del conflicto armado, aunque asociada de manera más significativa con las iniciativas personales, y mientras que los estudiantes de pregrado de Universidad Militar Nueva Granada deciden dedicarse principalmente a la actividad académica formal, los de la otra universidad muestran mayor interés por el desarrollo de acciones de servicio social o iniciativas personales, aunque no siempre la universidad sirva de plataforma para promover estas iniciativas ellos. La universidad pú- 
blica tiene la opción de reconocer estas iniciativas y apoyarlas mediante los diversos servicios con que cuenta, a fin de motivar a los estudiantes de pregrado en temas que son de su interés.

\section{Conclusiones}

El conflicto armado interno tiene repercusiones serias para los individuos que son afectados por las dinámicas de la violencia. Las decisiones adoptadas en el marco de la vida universitaria se encuentran limitadas al menos por dos factores, en el caso de los estudiantes víctimas del conflicto armado interno: 1) el impacto que se ha tenido en términos de pérdida de oportunidades y afectación a derechos y 2) las posibilidades que ofrece la universidad para desarrollar actividades tendientes a apoyar o acompañar personas con estas características. Sin embargo, estas decisiones se centran principalmente en el desarrollo de algunas actividades académicas o en la participación en proyectos o semilleros de investigación.

Los hallazgos de la investigación permiten identificar que el estudiante, al tomar el tipo de decisiones exploradas, es decir, al optar por las ayudas en investigación o en materias complementarias o al adelantar iniciativas de carácter personal, no lo hace por obtener algún beneficio o retribución, sino inscrito en un proceso que involucra toda su experiencia de vida para su satisfacción existencial y personal. De esta manera, la vida universitaria no solo está definida por los programas, estrategias y acciones que adelanta la universidad para los es- tudiantes, sino que además se materializa en las decisiones que adoptan los estudiantes respecto de su formación y sus acciones, las cuales se fundamentan en experiencias, emociones, imaginarios y perspectivas de vida, muchas de ellas trazadas en el marco del conflicto armado.

Sin embargo, la escasa difusión de las ayudas que ofrecen las universidades dificulta el acceso de los estudiantes a la participación en los programas de docencia, investigación y extensión, lo cual limita el reconocimiento de las reales problemáticas e impide la generación de nuevas ideas, estrategias y acciones que promuevan un verdadero cambio social y cultural. También es factible que los estudiantes de pregrado víctima del conflicto, por temor o por no ser identificados, decidan no participar, y otra posibilidad es que la oferta de servicios no sea atractiva o simplemente quieran centrarse en su actividad académica formal.

Dado lo anterior, y debido a que Bienestar Universitario no ha enfocado acciones específicas para aprovechar el potencial y las condiciones de los estudiantes víctima del conflicto armado con el objetivo de fomentar su movilización, se recomienda a las respectivas instituciones de educación superior identificar las principales características de estos grupos, a fin de adelantar estrategias de apoyo que no solo vinculen a los estudiantes que de una u otra forma han sido afectados, sino a otros actores, a fin de promover un aprendizaje colectivo de las experiencias y las realidades de otras personas. 


\section{Referencias}

Baquero, M. y Ariza, P. (2014). Educación, paz y posconflicto: oportunidades desde la educación superior. Revista de la Universidad de La Salle, 65, 115-134. Recuperado de https://ciencia.lasalle.edu.co/cgi/viewcontent. cgi?article $=1428 \&$ context $=$ ruls

Barreto, M. (2015). El programa de desarrollo y paz del Magdalena Medio, ¿un modelo de construcción de paz para el postconflicto en Colombia? Papel Político, 2O(2), 461-479. DOI: http://dx.doi.org/10.11144/Javeriana. papozO-2.pdpm

Cañadas, M. et al. (2006). Alerta 2006: informe sobre conflictos, derechos humanos y construcción de paz. Cataluña: Icaria.

Delgado, M. (2010). La universidad como constructora de paz: reflexiones conceptuales sobre la contribución de las universidades a la superación del conflicto. Revista de Análisis Internacional, 1, 119-140. Recuperado de https://expeditiorepositorio.utadeo.edu.co/handle/20.500.12010/898

De Zubiría, S. (2015). Dimensiones políticas y culturales en el conflicto colombiano. En: Comisión Histórica del Conflicto y sus Víctimas, Contribución al entendimiento del conflicto armado en Colombia. Bogotá: CHCV.

Díaz, A. M. y Sánchez, F. (2004). Geografía de los cultivos ilícitos y conflicto armado en Colombia. Documento CEDE 2004-18. Bogotá: Universidad de los Andes.

Echavarría, C., Bernal, J., Murcia, N., González, L. y Castro, L. (2015). Contribuciones de la institución educativa al postconflicto: Humanizarte, una propuesta pedagógica para la construcción de paz. Cuadernos de Administración, 28(51), 159-187. DOI: http://dx.doi.org/10.11144/Javeriana.cao28-51.ciep.

Elster, J. (1995). Tuercas y tornillos. Una introducción a los conceptos básicos de las ciencias sociales. Barcelona: Gedisa.

FIP - Fundación Ideas para la Paz (2015). Capacidades locales para la paz. Resultados y recomendaciones nacionales. Bogotá: FIP.

Fisas, V. (1998). Cultura de paz y gestión de conflictos (vol. 117). Barcelona: Icaria.

García, J. (2000). Lecciones de Metafísica Tomista. Navarra: Eunsa.

Giraldo, J. (2015). Aportes sobre el origen del conflicto armado en Colombia, su persistencia y sus impactos. En: Comisión Histórica del Conflicto y sus Víctimas, Contribución al entendimiento del conflicto armado en Colombia. Bogotá: CHCV.

Girard, K.y Koch, S. (2001). Resolución de conflictos en las escuelas: Manual para educadores. Barcelona: Granica.

Infante, A. (2013). El papel de la educación en situaciones de posconflicto: estrategias y recomendaciones. Hallazgos, 11(21), 223-245. DOI: https://doi.org/10.15332/s1794-3841.2014.0021.13

Larraín, A.y González, L. (2005). Formación universitaria por competencias. Santiago de Chile: Cinda. 
Miklos, T. (2001). Las decisiones politicas. De la planeación a la acción. Buenos Aires: Siglo XXI.

Murcia, N. (2009). Vida universitaria e imaginarios: posibilidad en definición de políticas sobre educación superior. Revista Latinoamericana de Ciencias Sociales, Niñez y Juventud, 7(1), 235-266. Recuperado de http:// www.redaly c.org/articulo.oa?id $=77307110$

Nasi, C. y Rettberg, A. (2005). Los estudios sobre conflicto armado y paz: un campo en evolución permanente. Colombia Internacional, 62, 64-85. DOI: https://doi.org/10.7440/colombiaint62.2005.04

Nieto, J. (2004). La guerra interna y el desplazamiento forzado. En: M. Nubia Bello (edit.), Desplazamiento forzado. Dinámicas de guerra, exclusión y desarraigo (pp. 31-48). Bogotá: Universidad Nacional de Colombia.

Novelli, M. y López, M. (2008). Conflict, education and the global south: new critical directions. International Journal of Educational Development, 28, 473-488. DOI: https://doi.org/10.1016/j.ijedudev.2008.01.004

Paulson, J. y Rappleye, J. (2007). Education and conflict: essay review. International Journal of Educational Development, 27, 340-347. DOI: http://dx.doi.org/10.1016/j.ijedudev.2006.10.010

Pérez, T. (2014). Colombia: de la educación en emergencia hacia una educación para el posconflicto y la paz. Revista Interamericana de Investigación, Educación y Pedagogía, 7(2), 287-311. DOI: https://doi.org/10.15332/ s1657-107X.2014.0002.06

Pizarro, E. (2015). Una lectura múltiple y pluralista de la historia. En: Comisión Histórica del Conflicto y sus Víctimas, Contribución al entendimiento del conflicto armado en Colombia. Bogotá: CHCV.

Restrepo, H. (2013). Repensando la universidad en su dimensión político-democrática para los escenarios de paz. IM-Pertinente, 1(1), 153-161.

Salm, R. (2006). La solución de conflictos en la escuela, una guía práctica para maestros. Bogotá: Cooperativa Editorial Magisterio.

Smith, D. (2000). Tendencias y causas del conflicto armado. Berghof Research Center for Constructive Conflict Management.

Torrijos, V. (2015). Cartografía del conflicto: pautas interpretativas sobre la evolución del conflicto irregular colombiano. En: Comisión Histórica del Conflicto y sus Víctimas, Informe final (pp. 652-696). Bogotá.

Trilla, J., Gonzalo, J., Martínez, M. y Romañá, T. (2011). La participación de los estudiantes en el gobierno y la vida universitaria. Encounters on Education, 12, 93-111. Recuperado de http://www.revistaeducacion.educacion. es/re358/re358_25.pdf

Vidal, G. (2008). La teoría de la elección racional en las ciencias sociales. Sociológica, 23(67), 221-236. Recuperado de http://www.sociologicamexico.azc.uam.mx/index.php/Sociologica/article/view/180

Zurbano, D. (1998). Bases de una educación para la paz y la convivencia. Navarra: Gobierno de Navarra/Departamento para la Educación y la Cultura. 